\title{
Rhinovirus infection liberates planktonic bacteria from biofilm and increases chemokine responses in cystic fibrosis airway epithelial cells
}

\author{
Sangbrita S Chattoraj, ${ }_{1}^{1}$ Shyamala Ganesan, ${ }^{1}$ Andrew M Jones, ${ }^{2}$ Jennifer M Helm, ${ }^{2}$ \\ Adam T Comstock, ${ }^{1}$ Rowland Bright-Thomas, ${ }^{2}$ John J LiPuma, ${ }^{1}$ Marc B Hershenson, ${ }^{1,3}$ \\ Umadevi S Sajjan ${ }^{1}$
}

\begin{abstract}
- Additional data are published online only. To view these files please visit the journal online at (http://thorax.bmj.com).

1 Department of Pediatrics and Communicable Diseases, University of Michigan, Ann Arbor, Michigan, USA

${ }^{2}$ Manchester Adult Cystic Fibrosis Center, University Hospitals South Manchester NHS Trust Wythenshawe Hospital, Manchester, UK ${ }^{3}$ Department of Molecular and Integrative Physiology, University of Michigan, Ann Arbor, Michigan, USA
\end{abstract}

\section{Correspondence to}

Umadevi S Sajjan, University of Michigan, 1150 W Medical Center Dr, Room 3570, MSRBII, Box 5688, Ann Arbor, MI USA; usajjan@umich.edu

Received 16 September 2010 Accepted 23 December 2010 Published Online First 2 February 2011

\section{ABSTRACT}

Background Intermittent viral exacerbations in patients with cystic fibrosis (CF) with chronic Pseudomonas aeruginosa (PA) infection are associated with increased bacterial load. A few clinical studies suggest that rhinoviruses (RV) are associated with the majority of viralrelated exacerbations in CF and require prolonged intravenous antibiotic treatment. These observations imply that acute RV infection may increase lower respiratory symptoms by increasing planktonic bacterial load. However, the underlying mechanisms are not known. Methods Primary CF airway epithelial cells differentiated into mucociliary phenotype were infected with mucoid PA (MPA) followed by RV and examined for bacterial density, biofilm mass, levels of chemokines and hydrogen peroxide $\left(\mathrm{H}_{2} \mathrm{O}_{2}\right)$. The need for dual oxidase 2, a component of NADPH oxidase, in RV-induced generation of $\mathrm{H}_{2} \mathrm{O}_{2}$ in CF cells was assessed using gene-specific siRNA.

Results Superinfection with RV increased chemokine responses in CF mucociliary-differentiated airway epithelial cells with pre-existing MPA infection in the form of biofilm. This was associated with the presence of planktonic bacteria at both the apical and basolateral epithelial cell surfaces. Further, RV-induced generation of $\mathrm{H}_{2} \mathrm{O}_{2}$ via dual oxidase 2 in CF cells was sufficient for dispersal of planktonic bacteria from the biofilm. Inhibition of NADPH oxidase reduced bacterial transmigration across mucociliary-differentiated CF cells and the interleukin-8 response in MPA- and RV-infected cells.

Conclusion This study shows that acute infection with RV liberates planktonic bacteria from biofilm. Planktonic bacteria, which are more proinflammatory than their biofilm counterparts, stimulate increased chemokine responses in CF airway epithelial cells which, in turn, may contribute to the pathogenesis of CF exacerbations.

Pulmonary manifestations due to chronic endobronchial infection are the leading cause of morbidity and mortality in individuals with cystic fibrosis (CF). Pseudomonas aeruginosa (PA), a principal pathogen in CF, chronically colonises the airways and often exists in a biofilm matrix. Despite chronic infection, patients with CF experience acute exacerbations only periodically, indicating that biofilm bacteria may act as a resilient reservoir for planktonic bacteria rather than a trigger for exacerbations. Consistent with this hypothesis, increased PA density was observed during episodes of acute exacerbations and required antibiotic treatment. ${ }^{1}$ Furthermore, PA isolated from sputum collected during exacerbations was similar to colonising flora by genotypic analysis, suggesting a clonal expansion of bacteria from the biofilm reservoir. ${ }^{2}$

The significance of respiratory viral infections in the pathogenesis of asthma and chronic obstructive pulmonary disease (COPD) exacerbations has long been recognised. Respiratory viral infection is implicated in $44-80 \%$ of asthma exacerbations and $46-50 \%$ of COPD exacerbations. ${ }^{3}$ Similarly, an improved method of viral detection suggested a significant role for viral infections in CF exacerbations. ${ }^{4-7}$ Respiratory viruses associated with CF exacerbations include influenza viruses $\mathrm{A} / \mathrm{B}$, rhinoviruses, respiratory syncytical viruses, parainfluenza viruses and adenoviruses.

Rhinoviruses (RV), which cause common cold, are also responsible for the majority of viral-related exacerbations in asthma and COPD. ${ }^{3}$ A few clinical studies have suggested that RV is also associated with the majority of viral-related exacerbations in $\mathrm{CF}^{4}{ }^{7-9}$ In one study, patients with $\mathrm{RV}$ infection required prolonged intravenous antibiotic treatment. ${ }^{10}$ In another study, RV infection was associated with increased use of antibiotics, prolonged hospitalisation and decline in lung function. ${ }^{8}$ Similarly, respiratory syncytial virus infection in patients with intermittent or chronic PA infection was associated with increased pseudomonal antibody levels. ${ }^{10}$ This circumstantial evidence suggests that viral infection may increase planktonic bacterial load which, in turn, may stimulate an intense inflammatory response and increase clinical symptoms that can be reduced by treatment with antibiotics. Consistent with this notion, in vitro studies suggested a more intense host inflammatory response to motile planktonic bacteria. ${ }^{1112}$ However, the mechanisms by which viral infections increase the density of planktonic bacteria are not well known.

In the present study we examined whether superinfection with RV liberates planktonic bacteria and increases chemokine responses of CF airway epithelial cells preinfected with mucoid PA (MPA). We also investigated the mechanisms by which RV causes dispersal of planktonic bacteria from biofilm.

\section{METHODS}

Full details are given in the online data supplement. 


\section{Rhinovirus}

Rhinovirus serotype 39 was purchased from ATCC (Manassas, Virginia, USA). Viral stocks were generated and the tissue culture infectious dose $\left(\mathrm{TCID}_{50}\right)$ was determined as described previously. ${ }^{13}$

\section{Bacteria and growth conditions}

A clinical isolate of MPA was grown in tryptic soy broth (BD Diagnostics, Sparks, Maryland, USA) and suspended in phosphate buffer solution (PBS).

\section{CF cell cultures and infection}

Primary CF airway epithelial cells were obtained from three patients with CF and grown either at the air-liquid interface to promote mucociliary differentiation or as monolayers. ${ }^{14}$ The use of CF bronchial segments was reviewed by the University of Michigan Institutional Review Board. Mucociliary-differentiated cells were sequentially infected with MPA (at multiplicity of infection of 0.01$)$ followed by RV $\left(1 \times 10^{6} \mathrm{TCID}_{50}\right)$, UV-irradiated RV or sham. Chemokine levels in the basolateral medium were determined by ELISA (R\&D Systems, Minneapolis, Minnesota, USA). In some experiments the cells were preincubated with diphenylene iodonium (DPI) or 1140W (both from Sigma-Aldrich, St Louis, Missouri, USA), as indicated in the Results section. Cells treated with $0.1 \%$ dimethyl sulfoxide or media served as controls. Levels of lactose dehydrogenase (an index of cytotoxicity) were measured in the basolateral medium or cell culture supernatants from CF cell monolayers using Cytotox ${ }^{96}$ non-radioactive cytotoxicity kit (Promega, Madison, Wisconsin, USA).

\section{Dispersal of biofilm}

MPA biofilms were formed on the pegs of the MBEC assay system (Innovotech, Edmonton, Alberta, Canada) as described elsewhere. ${ }^{15}$ Briefly, the device consists of 96 conical pegs attached to a plastic lid and rests on a 96-well plate containing bacterial suspension $\left(\mathrm{OD}_{600}\right.$ 0.01) diluted in Lauria-Bertani broth and incubated for $48 \mathrm{~h}$. The pegs with biofilm were rinsed with sterile PBS and exposed to conditioned media from CF airway epithelial cells for $30 \mathrm{~min}$. The pegs were washed and stained with $0.2 \%$ crystal violet. Crystal violet bound to pegs was eluted with methanol and the optical density was measured at $590 \mathrm{~nm}$ to quantify the biofilm mass.

\section{Measurement of hydrogen peroxide}

Hydrogen peroxide $\left(\mathrm{H}_{2} \mathrm{O}_{2}\right)$ produced from cells was measured using the Amplex red hydrogen peroxide/peroxidase kit (Invitrogen, Carlsbad, California, USA).

\section{Transfection of cells}

CF cells were transfected with dual oxidase 2 (Duox2) siRNA (Dharmacon, Lafayette, Colorado, USA) or non-targeting (NT) siRNA using Lipofectamine RNAiMAX (Invitrogen) by reverse transfection following the manufacturer's instructions. Reduction in expression of Duox2 was determined by real-time PCR in RV-infected cells using the forward primer 5 -AAC CTA AGC AGC TCA CAA CT-3' and reverse primer 5'-CAG AGA GCA ATG ATG GTG AT-3'. The specificity of the product was determined by melting curve analysis.

\section{Confocal microscopy}

Cultures were fixed in methanol and incubated with a mixture of antibodies to PA (Abcam, Cambridge, Massachusetts,
USA) and zona occludins (ZO)-1 (BD Biosciences, San Jose, California, USA).

\section{Scanning electron microscopy}

Cell cultures were processed for scanning electron microscopy as described previously. ${ }^{14}$

\section{Statistical analysis}

Results are expressed as mean \pm SEM or median (range). To compare groups, one-way analysis of variance (ANOVA) with Tukey-Kramer post hoc analysis or ANOVA based on ranks with Dunn's post hoc analysis was performed, as appropriate.

\section{RESULTS}

\section{Superinfection with RV increases chemokine responses in MPA-infected CF airway epithelial cells}

CF airway epithelial cells differentiated into mucociliary phenotype were infected with MPA or treated with PBS; $24 \mathrm{~h}$ later the cells were infected with sham, RV or replication-deficient UV-irradiated RV (UV-RV). Compared with cell cultures treated with PBS, cultures infected with MPA, RV or UV-RV showed significant increases in the chemokines interleukin 8 (IL-8), growth-regulated oncogene $\alpha(\mathrm{GRO}-\alpha)$ and epithelial cell-derived neutrophil-activating peptide 78 (ENA-78) (figure 1A-C). Cell cultures co-infected with MPA/RV showed synergistic increases in the levels of all three chemokines measured compared with cells infected with MPA or RV alone. This synergistic increase in chemokines was not observed in cells infected with MPA/UV-RV, indicating that an infectious virus is required for the observed increases.

Small increases in both bacterial and viral loads were observed in cells co-infected with MPA and RV compared with cells infected with MPA alone or MPA/UV-RV (see tables 1 and 2 in online supplement). Together, these results suggest that the observed synergistic or additive chemokine responses depend on other factors besides small increases in bacterial and/or viral load.

\section{RV infection facilitates transmigration of PA across mucociliary-differentiated CF airway epithelial cells}

Cells infected with MPA/UV-RV or MPA/RV were immunostained with antibody to $\mathrm{PA}$ and $\mathrm{ZO}-1$, a component of tight junction, and subjected to confocal indirect immunofluorescence microscopy. Basolateral media from these cultures were plated to determine the number of translocated bacteria. Confocal microscopy showed normal distribution of ZO-1 in the periphery of the cells and the presence of bacterial microcolonies (appearing as a green haze) with very few individual bacteria on the apical surface of MPA/UV-RV-infected cells (figure 2A). In contrast, MPA/RV-infected cell cultures showed dissociation of ZO-1 from the periphery of the cells in some areas and the presence of bacterial microcolonies along with numerous individual bacteria on the apical surface (figure 2B). Z-sections of these cultures revealed that, while bacteria were mainly on the apical surface in MPA/UV-RV-infected cultures (figure 2C), they were observed between the cells and at the basolateral surface in MPA/RV-infected cultures (figure 2D). Further, bacteria were recovered consistently $\left(4.2 \times 10^{4} \pm 1.6 \times 10^{4} \mathrm{CFU} /\right.$ $\mathrm{ml})$ in the basolateral media obtained from MPA/RV-infected cells, but not from MPA/UV-RV-infected cells. These results indicate that secondary infection with RV facilitates dispersal of bacteria from the microcolonies and transmigration of dispersed 
Figure 1 Rhinovirus (RV)

synergistically increases chemokine expression in cells infected with mucoid Pseudomonas aeruginosa (MPA). Mucociliary-differentiated cystic fibrosis (CF) airway epithelial cells were infected apically with MPA or treated with phosphate buffer solution (PBS) for $24 \mathrm{~h}$. The cells were then infected with RV and incubated for another $24 \mathrm{~h}$.

Sham or ultraviolet-irradiated

RV-infected cells (UV-RV) were used as controls. The following chemokine levels were measured in the basolateral medium: (A) interleukin 8 (IL-8); (B) growth-regulated oncogene $\alpha$ (GRO- $\alpha$ ); (C) epithelial cell-derived neutrophilactivating peptide 78 (ENA-78). Data represent median and range calculated from two independent experiments performed in duplicate on cells obtained from three CF donors. Numbers within each panel represent medians calculated from six replicates for MPA-, RV- or MPA/RV-infected cells to show the synergistic increases. ${ }^{*} p \leq 0.05$ vs PBS-treated group; $t p \leq 0.05$ vs all other treatment groups (ANOVA on ranks with Dunn's post hoc analysis).
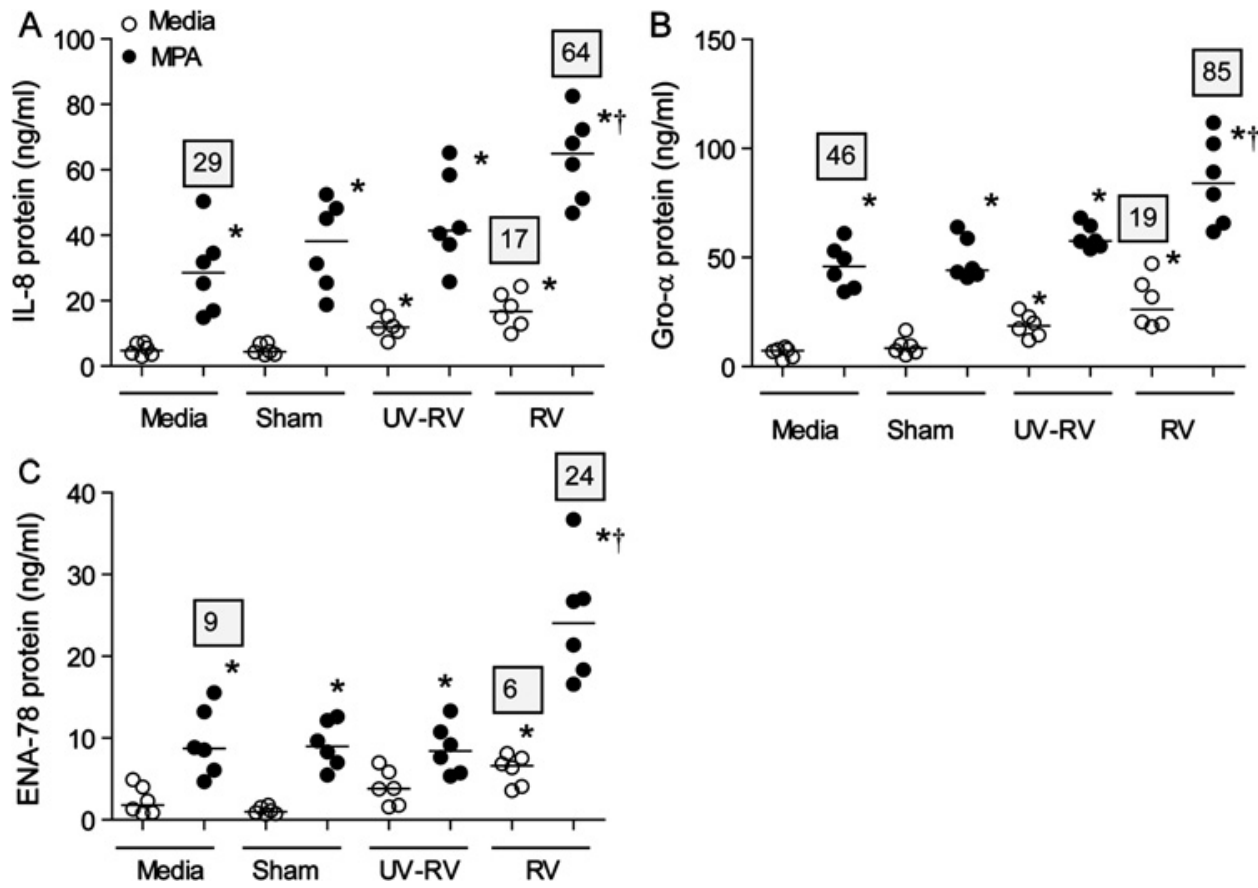

bacteria across the differentiated CF cultures, and this may potentially increase the interaction of bacteria with basolateral receptors.

\section{RV infection causes dispersal of bacteria from MPA biofilm}

Scanning electron microscopy of MPA/UV-RV-infected mucociliary-differentiated cell cultures revealed a film covering the majority of the bacteria, suggesting the presence of bacteria in a biofilm (figure $3 \mathrm{~A}$ ). In contrast, MPA/RV-infected cultures showed hollows in the biofilm which were surrounded by bacteria that were not covered by a film, an indication of dispersal of the bacteria from the biofilm (figure $3 \mathrm{~B}$ ). These results imply that $\mathrm{RV}$ infection can disperse planktonic bacteria from biofilm.

To determine whether RV is sufficient to disperse bacteria from biofilm, MPA biofilm grown on plastic pegs of an MBEC device (Innovotech) was exposed to PBS, cell culture media, RV inoculum equivalent to $1 \times 10^{6} \mathrm{TCID}_{50}$ or UV-RV for $3 \mathrm{~h}$ and the biofilm mass left on the peg was quantified. None of these treatments affected the biofilm mass (figure $3 \mathrm{C}$ ), suggesting that a factor(s) secreted by epithelial cells upon RV infection is responsible for dispersal of bacteria from biofilm. This led us to examine the effect of conditioned media from RV- or UV-RVinfected cells on biofilm density. In the subsequent experiments
Figure 2 Rhinovirus (RV) facilitates transmigration of mucoid Pseudomonas aeruginosa (MPA) across cystic fibrosis (CF) airway epithelial cells. Welldifferentiated CF airway epithelial cells were sequentially infected with MPA followed by ultraviolet-irradiated RV (UV-RV) $(A, C)$ or RV (B, D). Cells were immunostained with antibodies to a mixture of $P$ seudomonas aeruginosa (green) and zona occludin-1 (ZO-1; red) and counterstained with DAPI 4',6'-dimaino-2-phenylindole (blue). Images are representative of three independent experiments. (A, B) Apical view of cell cultures; (C, D) Z-section generated from the area marked with a white line in $(A)$ and $(B)$, respectively. Arrows in $(A)$ and $(B)$ represent bacterial microcolonies; arrowheads and asterisk in (B) represent two or three bacteria in a group and dissociation of ZO-1 from the periphery of cells, respectively.

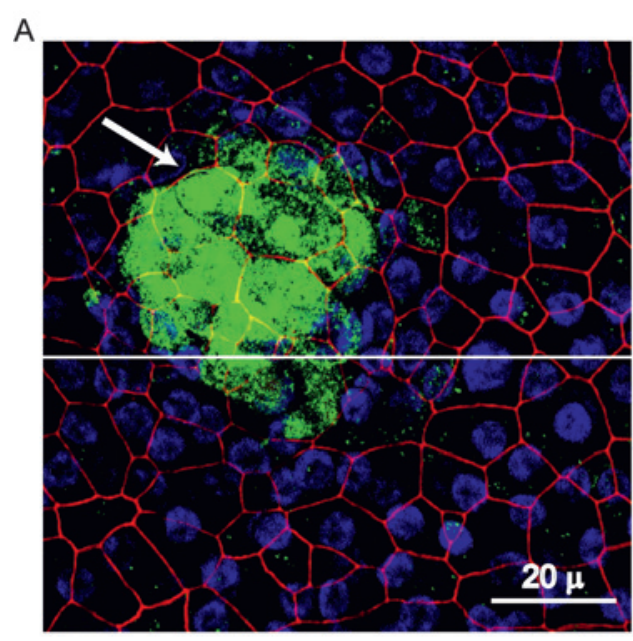

B
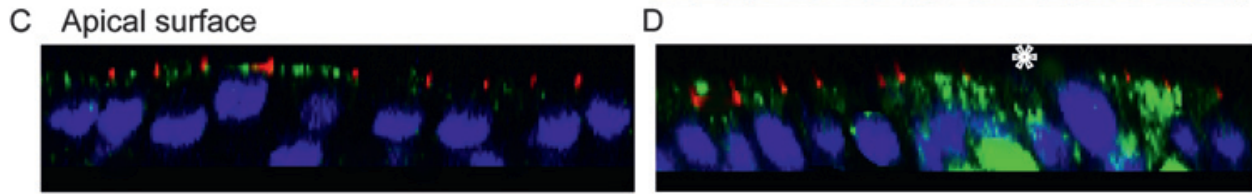
Figure 3 Rhinovirus (RV) disperses bacteria from mucoid Pseudomonas aeruginosa (MPA) biofilm. Welldifferentiated cystic fibrosis (CF) airway epithelial cells were sequentially infected with MPA followed by (A) ultraviolet-irradiated RV (UV-RV) or (B) RV and subjected to scanning electron microscopy. The asterisk and arrowhead represent MPA biofilm and individual bacteria; arrows in (B) point to hollows in the biofilm surrounded by bacteria not embedded in the biofilm, an indication of dispersed bacteria from biofilm. Images are representative of three independent experiments. (C) MPA biofilm grown on plastic pegs of the MBEC device were incubated with phosphate buffer solution, sham, RV inoculum or UV-RV and biofilm mass determined. (D) MPA biofilm grown on plastic pegs of the MBEC device were incubated with conditioned media from CF airway epithelial cells exposed to media, sham, RV or UV-RV and biofilm mass determined. Data represent mean \pm SEM calculated from four independent experiments performed in quadriplicate. ${ }^{*} p \leq 0.05$ vs conditioned media from cells exposed to media, sham and UV-RV (ANOVA with

Tukey-Kramer post hoc analysis).
A

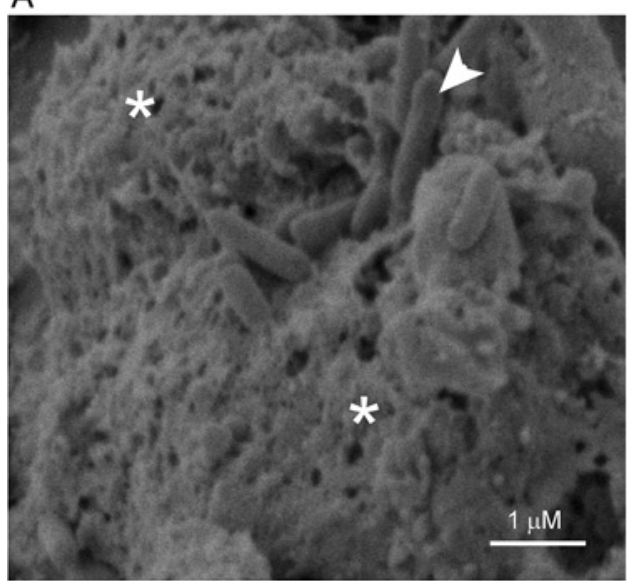

B
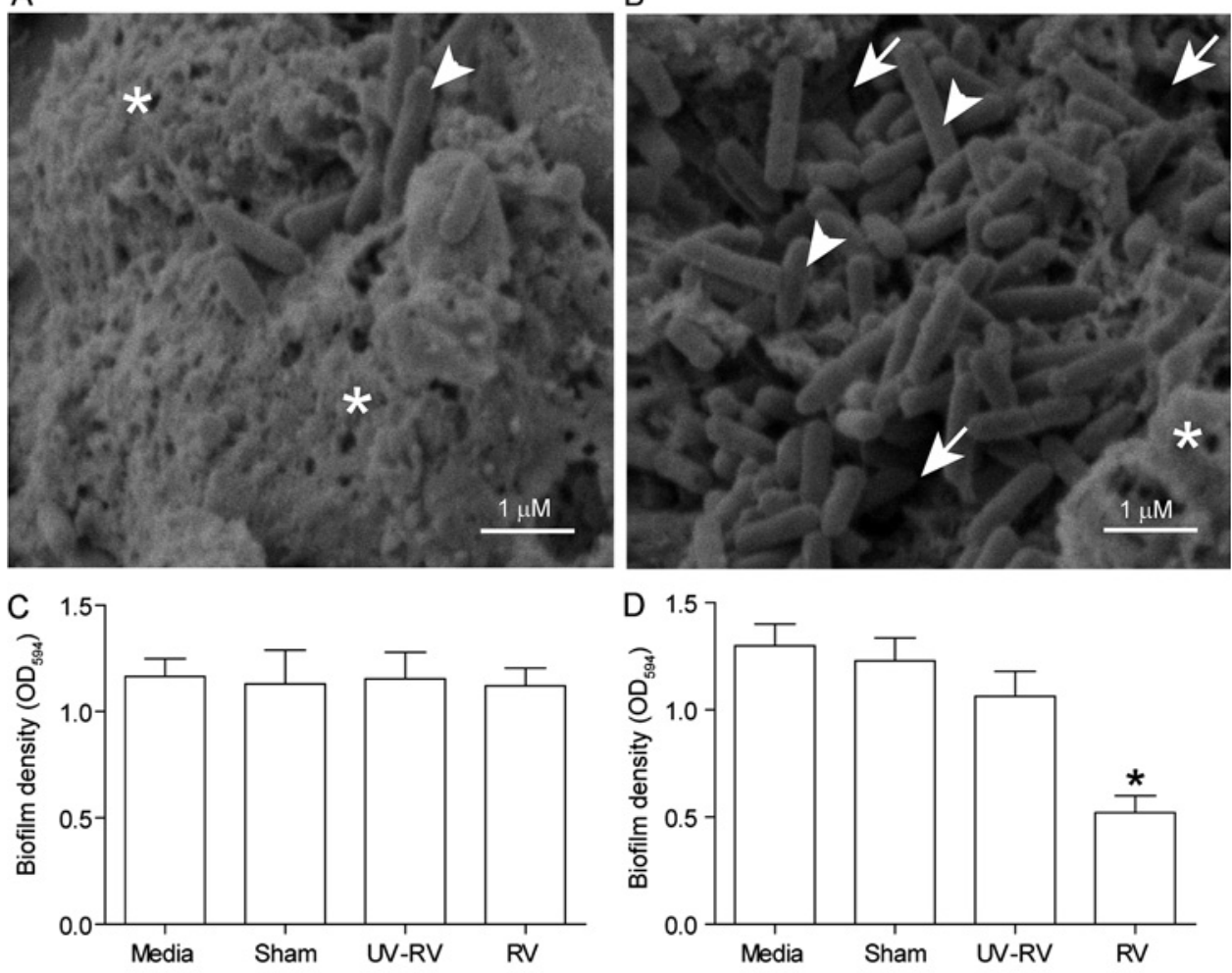

Conditioned media from CF cells monolayers of CF primary cells were used because mucus present on the apical surface of differentiated cells interfered with the assay. Exposure of MPA biofilm grown on plastic pegs to conditioned media from RV-infected but not from UV-RV-infected cells decreased biofilm density (figure 3D). These results indicate that a factor(s) released from CF cells in response to infectious RV is responsible for biofilm disruption.

\section{$R V$-induced $\mathrm{H}_{2} \mathrm{O}_{2}$ generation is sufficient for disruption of biofilm}

RV has been shown to induce oxidative stress, ${ }^{16}{ }^{17}$ and such environmental change can cause disruption of bacterial biofilm. ${ }^{18-20}$ We therefore examined the capacity of conditioned media from CF cells infected with RV or UV-RV in the presence of antioxidants to disrupt MPA biofilm. Treatment with DPI, an inhibitor of NADPH oxidase, completely reversed the capacity of conditioned media from RV-infected cells to disrupt biofilm (figure $4 \mathrm{~A}$ and online figure $1 \mathrm{~A}$ ). In contrast, conditioned media from cells infected with RV in the presence of $1140 \mathrm{~W}$, an inhibitor of inducible nitric oxide synthase, had no effect on biofilm mass (see online figure 1C). Neither DPI nor 1140W alone had an effect on biofilm mass. Also, these inhibitors did not affect cell viability or viral load in the cells (see online figure $1 \mathrm{E}$ and $1 \mathrm{~F}$ ). These results indicate that DPI-inhibitable oxidative stress caused by RV is responsible for dispersion of biofilm bacteria.

DPI inhibits flavoenzymes including NADPH oxidases. RV infection increased mRNA expression of Duox2, a component of $\mathrm{NADPH}$ oxidase, by $5.6 \pm 1.2$-fold compared with sham or UV-RV-infected cells. Duox2 generates and secretes $\mathrm{H}_{2} \mathrm{O}_{2}$ directly into the extracellular milieu. In addition, RV infection also stimulated the generation of $\mathrm{H}_{2} \mathrm{O}_{2}$ in CF airway epithelial cells $(12.6 \pm 5.81 \mu \mathrm{M})$, and this was inhibited by DPI (figure $4 \mathrm{~B}$ and online figure $1 \mathrm{~B})$. To determine whether Duox2 stimulated by $\mathrm{RV}$ infection is required for generation of $\mathrm{H}_{2} \mathrm{O}_{2}$, monolayers of CF cells were transfected with non-targeting (NT) siRNA or siRNA specific to Duox2. CF cells transfected with siRNA specific to Duox2, but not NT siRNA, blocked the expression of Duox2 (figure 4C and online figure $2 \mathrm{~A}$ and $2 \mathrm{~B}$ ) and inhibited $\mathrm{H}_{2} \mathrm{O}_{2}$ generation from $\mathrm{RV}$-infected cells (figure 4D). In addition, conditioned media from Duox2 siRNA infected with RV was attenuated in its capacity to disrupt biofilm (figure 4E). This was not due to change in RV load in Duox2 siRNA transfected cells as there was no difference in RV load between NT-siRNA and Duox2 siRNA transfected cells (online figure 2C).

To further confirm that $\mathrm{H}_{2} \mathrm{O}_{2}$ induced by $\mathrm{RV}$ contributes to biofilm disruption, we initially determined the effect of $\mathrm{H}_{2} \mathrm{O}_{2}$ on the biofilm mass grown on plastic pegs. $\mathrm{H}_{2} \mathrm{O}_{2}$ decreased the biofilm mass in a concentration-dependent manner (online figure 3). Catalase, which neutralises $\mathrm{H}_{2} \mathrm{O}_{2}$, inhibited the reduction of biofilm mass caused by both $\mathrm{H}_{2} \mathrm{O}_{2}$ and conditioned media from RV-infected cells (figure $4 \mathrm{~F}$ ). These results confirmed that $\mathrm{H}_{2} \mathrm{O}_{2}$ generation induced by $\mathrm{RV}$ is required for disruption of biofilm.

\section{DPI inhibits bacterial transmigration and IL-8 increase in differentiated CF cells superinfected with RV}

To assess whether $\mathrm{H}_{2} \mathrm{O}_{2}$ induced by $\mathrm{RV}$ is required for the bacterial transmigration and observed synergistic increases in IL-8 production, well-differentiated CF airway epithelial cells were infected with PBS or MPA and incubated for $24 \mathrm{~h}$. The cells were then infected with UV-RV or RV and incubated in the presence 0 or $5 \mu \mathrm{M}$ DPI and the basolateral media were examined for IL-8 levels and the presence of bacteria. As observed earlier, in the absence of DPI, MPA/RV-infected cultures showed synergistic increases in IL-8 (figure 5A) and bacteria in the basolateral media (figure $5 \mathrm{~B}$ ). In contrast, MPA/RV-infected cells 
Figure 4 Generation of hydrogen peroxide $\left(\mathrm{H}_{2} \mathrm{O}_{2}\right)$ in cystic fibrosis (CF) airway epithelial cells in response to rhinovirus (RV) infection is responsible for bacterial dispersal from biofilm. (A) Conditioned media from monolayers of CF airway epithelial cells infected with ultraviolet-irradiated RV (UV-RV) or RV in the presence of $0(-)$ or $5 \mu \mathrm{M}(+)$ diphenylene iodonium (DPI) were incubated with mucoid Pseudomonas aeruginosa (MPA) biofilm and the biofilm mass was determined. (B) CF airway epithelial cells infected with UV-RV or RV or treated with media in the presence of DPI (0 or $5 \mu \mathrm{M})$ and $\mathrm{H}_{2} \mathrm{O}_{2}$ present in the media was measured. (C) CF cells were transfected with non-targeting (NT) or dual oxidase 2 (Duox2) siRNA, infected with RV or UV-RV and expression of Duox2 was determined by immunoblot analysis, representative of three independent experiments. (D) Levels of $\mathrm{H}_{2} \mathrm{O}_{2}$ in media from monolayers of $\mathrm{CF}$ cells transfected with siRNA specific to Duox2 or NT siRNA and infected with UV-RV or RV. (E) MPA biofilm grown on plastic pegs was exposed to conditioned media from cells transfected with Duox2 siRNA or NT siRNA followed by UV-RV or RV infection and biofilm mass was quantified. Cells treated with media served as control. (F) MPA biofilm was exposed to conditioned media from RV-infected cells or $\mathrm{H}_{2} \mathrm{O}_{2}(10 \mu \mathrm{M})$ in the presence or absence of catalase and biofilm mass was measured. Data represent mean \pm SEM calculated from four independent experiments performed in duplicates or quadriplicates. ${ }^{*} p \leq 0.05$ vs control; $+p<0.05$ vs RV-infected group in the absence of DPI or NT siRNA-transfected cells infected with RV (ANOVA with Tukey-Kramer post hoc analysis).

C
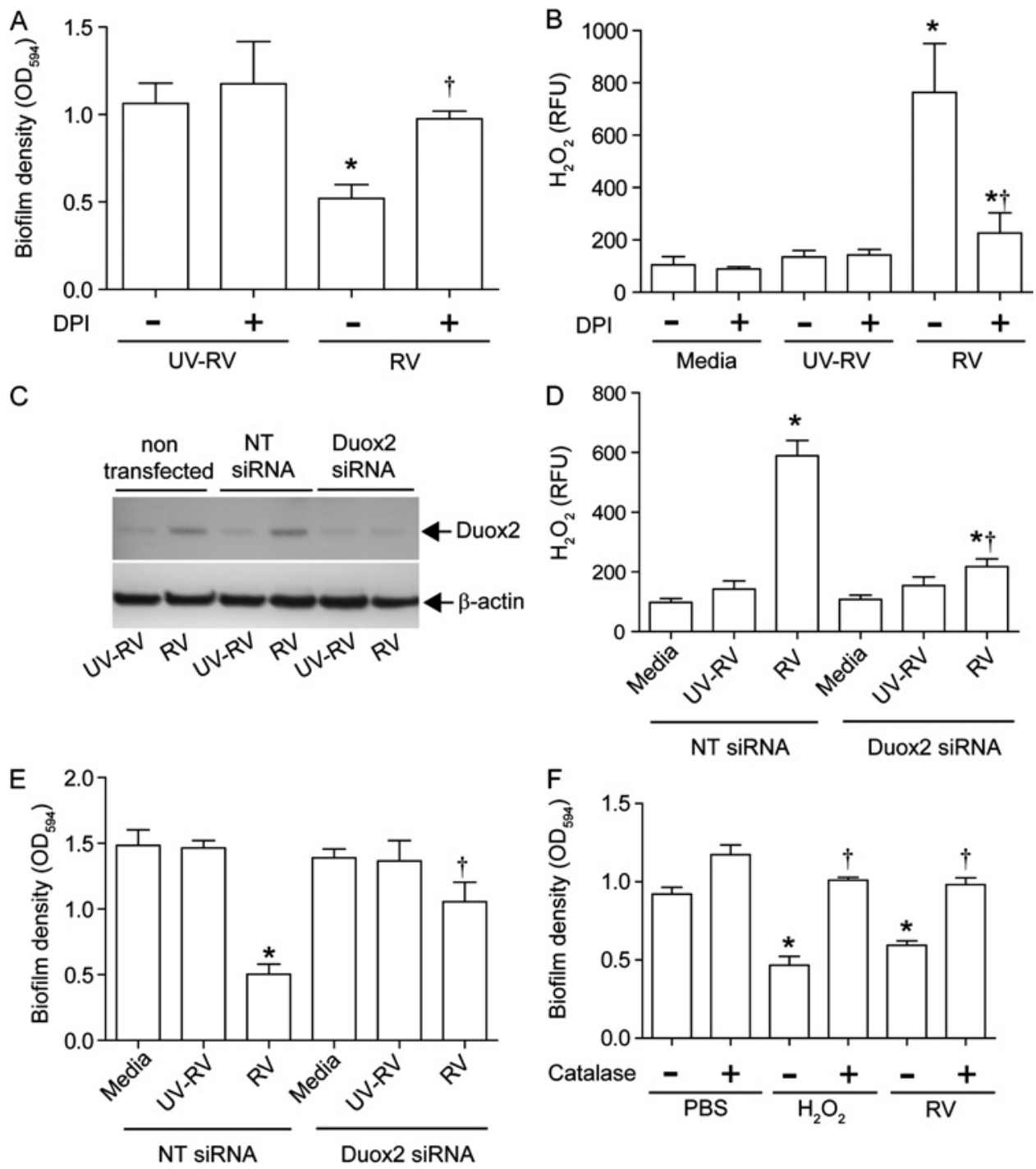

\section{DISCUSSION}

In this study we show that superinfection with RV stimulates robust chemokine responses from CF airway epithelial cells preinfected with MPA. We also show that increased cytokine responses are associated with RV-facilitated dispersal of bacteria from MPA biofilm and transmigration of planktonic bacteria from the apical to the basolateral surface of mucociliary-differentiated CF airway epithelial cells. Furthermore, we provide evidence that $\mathrm{RV}$ infection induces $\mathrm{H}_{2} \mathrm{O}_{2}$ production in $\mathrm{CF}$ airway epithelial cells and this in turn causes dispersal of planktonic bacteria from biofilm. Together these results suggest that acute superinfection with RV increases chemokine responses and planktonic bacteria by causing dispersal of biofilm.

RV stimulates oxidative stress and chemokine responses from airway epithelial cells. ${ }^{16}{ }^{21-23}$ It is therefore plausible that an $\mathrm{RV}$-induced subtle change in the microenvironment of CF airways is sufficient to increase the pathogenesis of existing flora. Consistent with this notion, RV-induced mild oxidative stress appears to be sufficient for liberation of planktonic bacteria from the biofilm. Planktonic bacteria are motile and express virulence factors, and hence can stimulate inflammatory responses readily compared with relatively immotile biofilm bacteria. ${ }^{12}$ In CF airways, MPA persists in biofilm and is less stimulatory to airway mucosa due to 'sandwich binding'. ${ }^{24}$ 
Figure 5 Diphenylene iodonium (DPI) inhibits bacterial transmigration and reduces interleukin 8 (IL-8) response in mucociliary-differentiated cultures sequentially infected with mucoid Pseudomonas aeruginosa (MPA) followed by rhinovirus (RV). Mucociliary-differentiated cystic fibrosis (CF) cultures were infected with MPA; $24 \mathrm{~h}$ later the cultures were infected with sham, ultravioletirradiated RV (UV-RV) or RV in the presence of DPI ( 0 or $5 \mu \mathrm{M}$ ) for another $24 \mathrm{~h}$. (A) IL-8 concentration in the basolateral media determined by ELISA. (B) Number of bacteria in the basolateral chamber determined by plating. Data represent mean \pm SEM or median with range from three independent experiments performed in duplicate ${ }^{*} p \leq 0.05$ vs media- or UV-RV-treated control groups; ${ }^{* *} p \leq 0.05$ vs respective RV-infected group, ANOVA with Tukey-Kramer post hoc analysis or ANOVA on ranks with Dunn's post hoc analysis) $\dagger-p<0.05$ vs all other groups. (C-F) Representative confocal images of cell cultures infected with MPA/RV in the presence of 0 or $5 \mu \mathrm{M} \mathrm{DPI}$,

respectively, showing bacteria (green), zona occludin-1 (red) and nuclei (blue). $(C, D)$ Apical view of cell cultures. $(E, F)$ Z-sections generated from an area marked with white lines in $(E)$ and $(F)$, respectively.

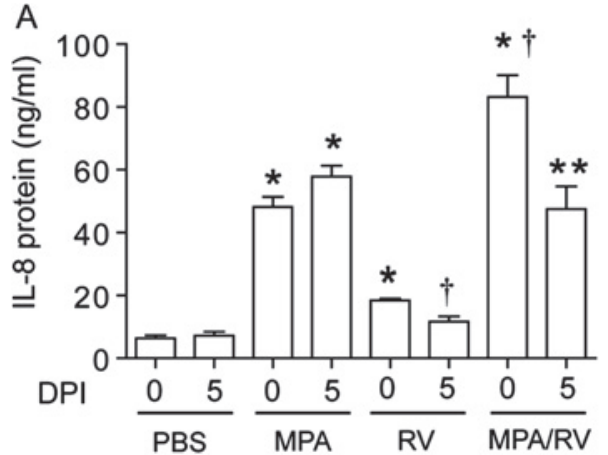

C

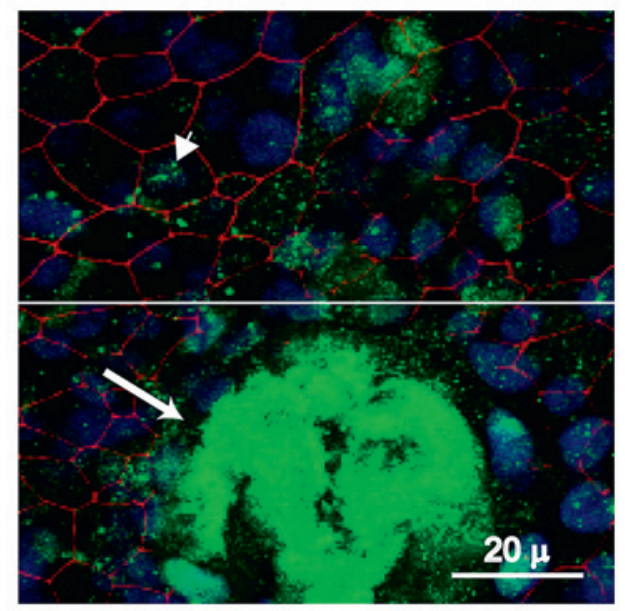

E

Apical Surface

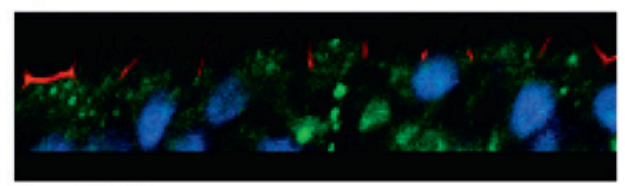

Basal Surface

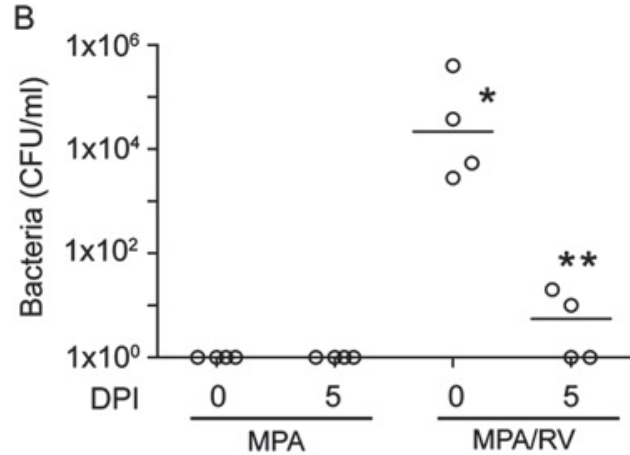

D

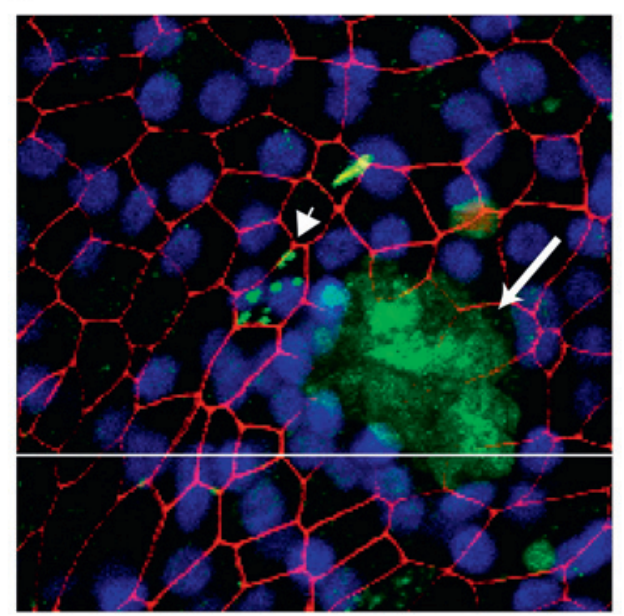

F

\section{Apical Surface}

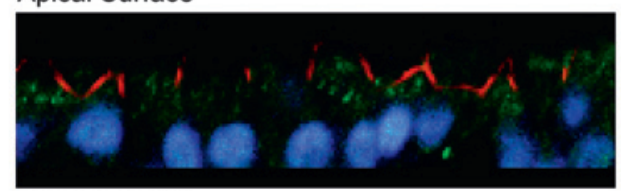

Basal Surface
Dispersal of planktonic bacteria from the biofilm caused by $\mathrm{RV}$ infection may therefore lead to increased chemokine responses.

Following exposure to oxidative or other environmental stress, bacteria within the biofilm undergo coordinated dispersal events releasing free-swimming planktonic bacteria. ${ }^{18-20} \mathrm{RV}$, which can stimulate the expression of nitric oxide (NO) and $\mathrm{H}_{2} \mathrm{O}_{2},{ }^{16} 172225$ therefore can disperse planktonic bacteria from the biofilm. In the present study we found that RV-stimulated $\mathrm{H}_{2} \mathrm{O}_{2}$ mediated by Duox2, but not $\mathrm{NO}$, is responsible for RV-induced liberation of planktonic bacteria from biofilm. Duox2 is one of the six homologues of NADPH oxidase catalytic phox subunit gp91 phox 26 and bronchial epithelial cells express Duox2 in response to treatment with Th1 and Th2 cytokines, TLR3 agonist or infection with $\mathrm{RV}^{27}$ Duox2 is located in the plasma membrane and can secrete $\mathrm{H}_{2} \mathrm{O}_{2}$ directly into the extracellular milieu, ${ }^{26} 28$ and thus can aid dispersal of bacteria from biofilm. Consistent with this notion, either inhibition of Duox2 expression or catalase, which neutralises the $\mathrm{H}_{2} \mathrm{O}_{2}$, abrogated the capacity of conditioned media from RV-infected cells to disrupt biofilm, suggesting that Duox2-mediated $\mathrm{H}_{2} \mathrm{O}_{2}$ generation plays a role in the dispersal of planktonic bacteria from the biofilm by RV. Lack of NO generation in CF cells in response to $\mathrm{RV}$ infection may be due to inducible nitric oxide synthase deficiency in these cells. ${ }^{29} 30$
In addition to dispersing bacteria from the biofilm, RV can also facilitate transmigration of bacteria across the airway epithelium because it has the capacity to compromise barrier function. ${ }^{31} 32$ This in turn may promote new interactions of dispersed planktonic bacteria with basolateral receptors which are otherwise not accessible, leading to increased production of cytokines from airway epithelial cells.

It is well established that bacteria in biofilms are more resistant to antibiotics than planktonic bacteria. ${ }^{33}$ In this regard, one can argue that disrupting biofilms to liberate planktonic bacteria that are more easily killed by antibiotics should be beneficial in CF where bacteria are thought to persist in biofilm. In addition, RVinduced $\mathrm{H}_{2} \mathrm{O}_{2}$ should also be beneficial in CF because $\mathrm{H}_{2} \mathrm{O}_{2}$ treatment has been proposed to kill planktonic bacteria. ${ }^{20}$ However, planktonic bacteria dispersed from biofilm are often as resistant to antibiotics as their biofilm counterparts ${ }^{34}$ and also express antioxidant enzymes such as alkyl peroxidase and catalase, each of which can neutralise $\mathrm{H}_{2} \mathrm{O}_{2}$ in the extracellular milieu. ${ }^{35}$ It is therefore conceivable that $\mathrm{RV}$-mediated dispersal of planktonic bacteria from biofilm increases inflammation in CF, requiring prolonged hospitalisation and use of intravenous antibiotics.

In conclusion, our results suggest that RV-induced oxidative stress liberates planktonic bacteria from biofilm and facilitates transmigration of planktonic bacteria across the mucociliarydifferentiated CF airway epithelial cells. This in turn may 
increase the interaction of planktonic bacteria with the basolateral receptors, leading to increased production of chemokines. These findings provide a novel mechanism by which RV infection may increase planktonic bacterial load and chemokine levels in CF.

Acknowledgements The authors thank Sasha Meshinchi, Microscopy and Image Analysis Laboratory, University of Michigan, for his assistance with scanning electron microscopy.

Funding This work was supported by National Institutes of Health grants HL0897720 (USS), HL082550 and HL081420 (MBH) and the Cystic Fibrosis Foundation (USS).

Competing interests None.

Ethics approval Use of CF bronchial segments was reviewed by the University of Michigan Institutional Review Board (IRB number HUM00000230).

Contributors SSC and SG conducted the study and analysed the data; ATM provided technical support; AMJ, JHM, RB, JJL and MBH helped to interpret data; JJL and MBH participated in critical review of manuscript; US and MBH obtained the funding; US designed the study, supervised its conduct and wrote the manuscript.

Provenance and peer review Not commissioned; externally peer reviewed.

\section{REFERENCES}

1. Goss $\mathbf{C H}$, Burns JL. Exacerbations in cystic fibrosis. 1: epidemiology and pathogenesis. Thorax 2007:62:360-7.

2. Aaron SD, Ramotar K, Ferris W, et al. Adult cystic fibrosis exacerbations and new strains of Pseudomonas aeruginosa. Am J Respir Crit Care Med 2004;169:811-15

3. Contoli M, Marku B, Conti V, et al. Viral infections in exacerbations of asthma and chronic obstructive pulmonary disease. Minerva Med 2009;100:467-78.

4. de Almeida MB, Zerbinati RM, Tateno AF, et al. Rhinovirus $C$ and respiratory exacerbations in children with cystic fibrosis. Emerg Infect Dis 2010;16:996-9.

5. van Ewijk BE, van der Zalm MM, Wolfs TF, et al. Prevalence and impact of respiratory viral infections in young children with cystic fibrosis: prospective cohort study. Pediatrics 2008;122:1171-6.

6. Wat D, Doull I. Respiratory virus infections in cystic fibrosis. Paediatr Respir Rev 2003:4:172-7.

7. Wat D, Gelder C, Hibbitts $\mathrm{S}$, et al. The role of respiratory viruses in cystic fibrosis. $J$ Cyst Fibros 2008;7:320-8.

8. Collinson J, Nicholson KG, Cancio E, et al. Effects of upper respiratory tract infections in patients with cystic fibrosis. Thorax 1996;51:1115-22.

9. Smyth AR, Smyth RL, Tong CY, et al. Effect of respiratory virus infections including rhinovirus on clinical status in cystic fibrosis. Arch Dis Child 1995; 73:117-20.

10. Petersen NT, Hoiby N, Mordhorst $\mathrm{CH}$, et al. Respiratory infections in cystic fibrosis patients caused by virus, Chlamydia and Mycoplasma-possible synergism with Pseudomonas aeruginosa. Acta Paediatr Scand 1981;70:623-8.

11. Cobb LM, Mychaleckyj JC, Wozniak DJ, et al. Pseudomonas aeruginosa flagellin and alginate elicit very distinct gene expression patterns in airway epithelial cells: implications for cystic fibrosis disease. J Immunol 2004;173:5659-70.

12. Starkey M, Hickman JH, Ma L, et al. Pseudomonas aeruginosa small-colony variants have adaptations that likely promote persistence in the cystic fibrosis lung. J Bacteriol 2009;191:3492-503.

13. Sajjan US, Jia Y, Newcomb DC, et al. H. influenzae potentiates airway epithelial cell responses to rhinovirus by increasing ICAM-1 and TLR3 expression. FASEB J 2006:20:2121-3.
14. Sajjan U, Keshavjee S, Forstner J. Responses of well-differentiated airway epithelia cell cultures from healthy donors and patients with cystic fibrosis to Burkholderia cenocepacia infection. Infect Immun 2004;72:4188-99.

15. Ceri H, Olson ME, Stremick C, et al. The Calgary Biofilm Device: new technology for rapid determination of antibiotic susceptibilities of bacterial biofilms. J Clin Microbiol 1999:37:1771-6

16. Kaul P, Biagioli MC, Singh I, et al. Rhinovirus-induced oxidative stress and interleukin-8 elaboration involves p47-phox but is independent of attachment to intercellular adhesion molecule-1 and viral replication. J Infect Dis 2000; 181:1885-90.

17. Biagioli MC, Kaul P, Singh I, et al. The role of oxidative stress in rhinovirus induced elaboration of IL-8 by respiratory epithelial cells. Free Radic Biol Med 1999;26:454-62

18. Sauer K, Cullen MC, Rickard AH, et al. Characterization of nutrient-induced dispersion in Pseudomonas aeruginosa PA01 biofilm. J Bacteriol 2004; 186:7312-26

19. Gjermansen $\mathbf{M}$, Ragas $\mathbf{P}$, Sternberg $\mathbf{C}$, et al. Characterization of starvation-induced dispersion in Pseudomonas putida biofilms. Environ Microbiol 2005;7:894-906.

20. Barraud N, Hassett DJ, Hwang SH, et al. Involvement of nitric oxide in biofilm dispersal of Pseudomonas aeruginosa. J Bacteriol 2006;188:7344-53.

21. Newcomb DC, Sajjan U, Nanua S, et al. Phosphatidylinositol 3-kinase is required for rhinovirus-induced airway epithelial cell interleukin-8 expression. J Biol Chem 2005:280:36952-61.

22. Papi A, Contoli M, Gasparini P, et al. Role of xanthine oxidase activation and reduced glutathione depletion in rhinovirus induction of inflammation in respiratory epithelial cells. J Biol Chem 2008;283:28595-606.

23. Spurrell JC, Wiehler S, Zaheer RS, et al. Human airway epithelial cells produce IP-10 (CXCL10) in vitro and in vivo upon rhinovirus infection. Am J Physiol Lung Cell Mol Physiol 2005;289:L85-95.

24. Kobayashi H. Airway biofilms: implications for pathogenesis and therapy of respiratory tract infections. Treat Respir Med 2005:4:241-53.

25. Sanders SP, Siekierski ES, Richards SM, et al. Rhinovirus infection induces expression of type 2 nitric oxide synthase in human respiratory epithelial cells in vitro and in vivo. J Allergy Clin Immunol 2001;107:235-43.

26. Lambeth JD. NOX enzymes and the biology of reactive oxygen. Nat Rev Immuno 2004:4:181-9.

27. Harper RW, Xu C, Eiserich JP, et al. Differential regulation of dual NADPH oxidases/ peroxidases, Duox1 and Duox2, by Th1 and Th2 cytokines in respiratory tract epithelium. FEBS Lett 2005:579:4911-17.

28. Geiszt M, Witta J, Baffi J, et al. Dual oxidases represent novel hydrogen peroxide sources supporting mucosal surface host defense. FASEB $J$ 2003;17:1502-4.

29. Meng $\mathbf{O H}$, Springall DR, Bishop AE, et al. Lack of inducible nitric oxide synthase in bronchial epithelium: a possible mechanism of susceptibility to infection in cystic fibrosis. J Pathol 1998;184:323-31.

30. Moskwa P, Lorentzen D, Excoffon KJ, et al. A novel host defense system of airways is defective in cystic fibrosis. Am J Respir Crit Care Med 2007;175:174-83.

31. Sajjan U, Wang 0 , Zhao $Y$, et al. Rhinovirus disrupts the barrier function of polarized airway epithelial cells. Am J Respir Crit Care Med 2008;178:1271-81.

32. Yeo NK, Jang YJ. Rhinovirus infection-induced alteration of tight junction and adherens junction components in human nasal epithelial cells. Laryngoscope 2010;120:346-52.

33. Hassett DJ, Korfhagen TR, Irvin RT, et al. Pseudomonas aeruginosa biofilm infections in cystic fibrosis: insights into pathogenic processes and treatment strategies. Expert Opin Ther Targets 2010;14:117-30.

34. Spoering $\mathbf{A L}$, Lewis K. Biofilms and planktonic cells of Pseudomonas aeruginosa have similar resistance to killing by antimicrobials. J Bacteriol 2001;183:6746-51.

35. Panmanee W, Hassett DJ. Differential roles of OxyR-controlled antioxidant enzymes alkyl hydroperoxide reductase (AhpCF) and catalase (KatB) in the protection of Pseudomonas aeruginosa against hydrogen peroxide in biofilm vs. planktonic culture. FEMS Microbiol Lett 2009;295:238-44. 